\title{
Medical Image of the Week: Infected Emphysematous Bulla
}

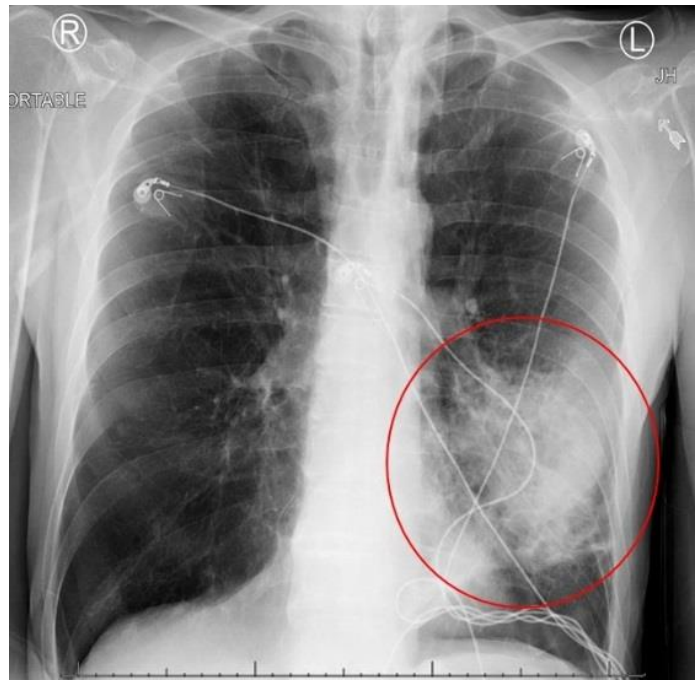

Figure 1. Portable AP chest X-ray revealing dense opacity within the lingula of left lung.

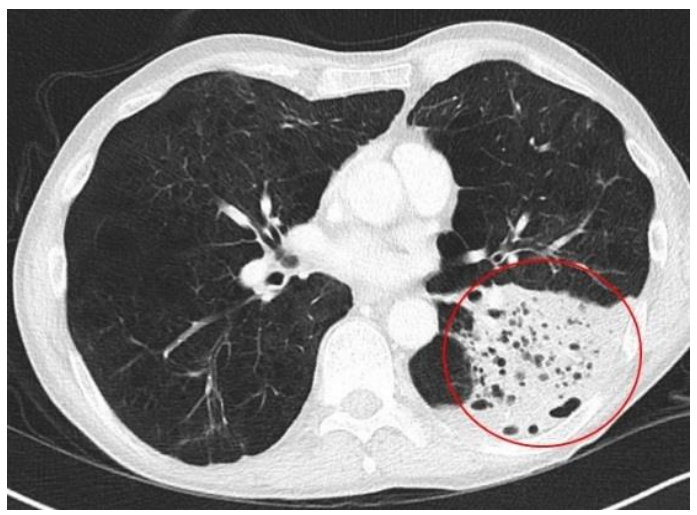

Figure 2. Thoracic CT with contrast showing lobar consolidation with increased lucency compatible with emphysema.

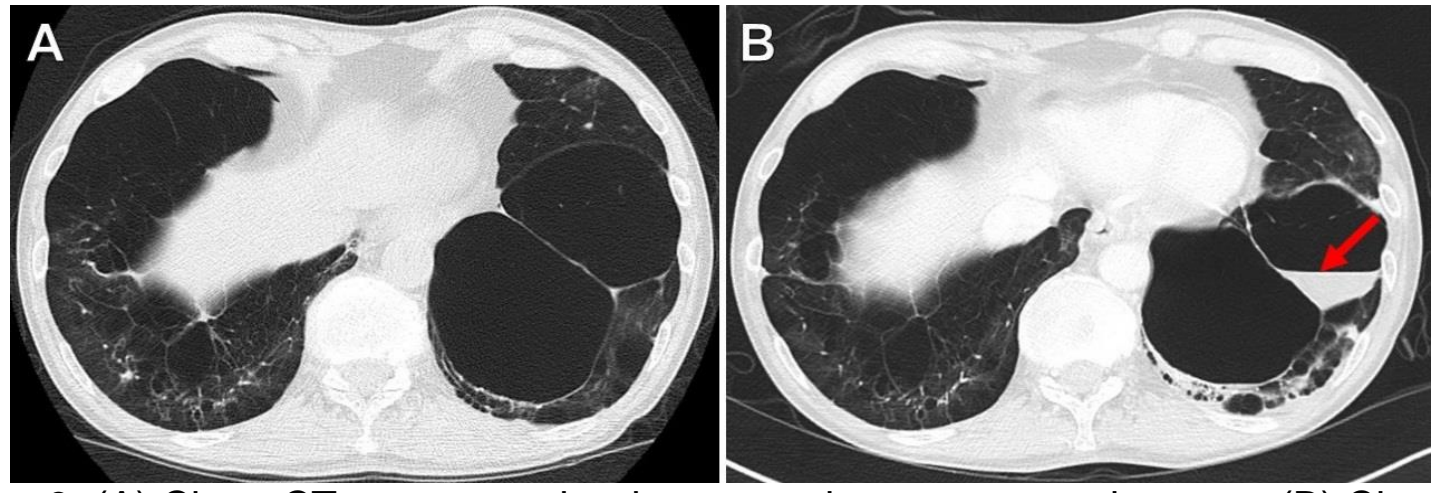

Figure 3. (A) Chest CT one year prior demonstrating severe emphysema. (B) Chest CT on admission showing new fluid-filled bulla (red arrow) in the setting pneumococcal pneumonia. 
A 65 year-old man with chronic obstructive lung disease (COPD), hypertension and alcohol abuse presented to the emergency department with complaints of feeling unwell and shortness of breath. He was tachycardic but otherwise hemodynamically stable, afebrile, and requiring 3 liters/min supplemental oxygen. Pertinent initial laboratory findings revealed a neutrophilic predominant leukocytosis (WBC $37.8 \times 10^{3} \mathrm{micro} / \mathrm{L}$ ) with lactic acidosis $(2.7 \mathrm{mMol} / \mathrm{L})$. Chest radiograph showed a dense opacity within the region of the lingula (Figure 1). Follow-up CT chest confirmed a consolidation likely representing lobar pneumonia in the setting of severe bullous emphysema (Figure 2). A large fluid-containing emphysematous bulla (Figure 3) was present which was not visualized one year prior.

He was started on broad spectrum antibiotics after peripheral blood cultures were drawn which revealed Streptococcus pneumoniae. Broad spectrum antibiotics were discontinued and patient was started on intravenous ceftriaxone $2 \mathrm{~g}$ every 24 hours. He improved clinically and was discharged home after 4 days.

Pneumococcal pneumonia remains the most common cause of community-acquired pneumonia and accounts for nearly $66 \%$ of all bacteremic pneumonias $(1,2)$. Our patient had multiple risk factors for developing pneumococcal pneumonia including alcohol abuse, COPD, and history of cigarette smoking. Pneumococcal pneumonia often causes dense consolidation within the lung in a well-defined lobar or segmental distribution. In emphysema areas of lucency may be seen within the consolidation which may mimic other processes such as necrosis. The pathogenesis of fluid accumulation in an emphysematous bulla is not well understood but can be associated with severe lung infection (3). Percutaneous drainage is not recommended and bronchoscopy is not usually required unless there is another indication (3). Antibiotics in those who are asymptomatic has not shown to add any benefit in resolution or preventing infection (3).

Norman Beatty MD¹, Kyle McKeown $\mathrm{MPH}^{2}$, Kelly M. Hager $\mathrm{MPH}^{2}$, and Stephen J. Scholand $\mathrm{MD}^{3}$

${ }^{1}$ Department of Medicine, Banner-University Medical Center South, Tucson, AZ USA

2 University of Arizona College of Medicine, Tucson, AZ USA

${ }^{3}$ Division of Infectious Diseases, Department of Medicine, MidState Medical Center, Meriden, CT USA

\section{References}

1. Torres A, Peetermans WE, Viegi G, Blasi F. Risk factors for community-acquired pneumonia in adults in Europe: a literature review. Thorax. 2013 Nov;68(11):105765. [CrossRef] [PubMed]

2. Fine MJ, Smith MA, Carson CA, Mutha SS, Sankey SS, Weissfeld LA, Kapoor WN. Prognosis and outcomes of patients with community-acquired pneumonia. A metaanalysis. JAMA. 1996 Jan 10;275(2):134-41. [CrossRef] [PubMed]

3. Chandra D, Rose SR, Carter RB, Musher DM, Hamill RJ. Fluid-containing emphysematous bullae: a spectrum of illness. Eur Respir J. 2008 Aug;32(2):303-6. [CrossRef] [PubMed] 